\title{
SHANGHAI, ¿SURPRISE?: CINCO ACTOS EN LA CONSTRUCCIÓN DE LA CIUDAD DEL PATRIMONIO EN CHINA
}

\author{
Plácido GonzÁlez Martínez \\ Professor. College of Architecture and Urban Planning. Tongji University, China \\ https://dx.doi.org/10.12795/astragalo.2017.i23.07
}

\section{INTRODUCCIÓN}

Ha pasado más de un cuarto de siglo desde que Saskia Sassen (1991) señalase hacia Londres, Tokio y Nueva York como nuevas ciudades globales, selecto grupo en el cual las grandes metrópolis chinas buscan incluirse de acuerdo a su nuevo rol como motores del desarrollo económico mundial. Beijing, Shanghai, Shenzhen, Guangdong y Chengdu rivalizan a nivel nacional e internacional con el objetivo de posicionarse en este sentido (Yulong y Hammett, 2002), gracias a los recursos que las autoridades nacionales y locales ponen a su disposición, movilizando desde el planeamiento urbano a las políticas culturales (Timberlake et al., 2014).

La entrada de China en la Organización Mundial del Comercio en 2001 animó al gobierno nacional a poner en práctica un programa de promoción cultural, que con la apertura de los primeros Institutos Confucio en 2004 ha llevado a un aumento del soft power de la civilización china a nivel global (Rossiter, 2006). Lejos de buscar rivalizar con la hegemonía anglosajona, su objetivo último era el cambio del sistema productivo, desde el sector industrial, hacia una nueva sociedad de servicios y a una economía del conocimiento (Ren, Sun, 2012).

El turismo y las industrias culturales se han convertido en nuevos impulsores del desarrollo de lo que este artículo define como ciudad del patrimonio; producto derivado de la ciudad global, caracterizado por la inversión en el capital cultural que ya definió Bordieu (1986). Una de sus manifestaciones más claras reside en la confianza que la ciudad deposita en el patrimonio urbano, y en su potencial para atraer visitantes y contribuir a la creación de 
una imagen global atractiva. En este sentido, las autoridades locales de ciudades como Beijing y Shanghai han insistido en sus perfiles turísticos y creativos a nivel internacional con el apoyo de instituciones como UNESCO, cuyo renombre internacional contribuye a legitimar el discurso oficial, definitivamente entendido como discurso patrimonial autorizado en los términos planteados por Smith (2006).

Sirva de muestra cómo Beijing cuenta en la actualidad con siete lugares inscritos en la Lista de Patrimonio Mundial (LPM), más que cualquier otra ciudad en el mundo, y a la espera de confirmar la inclusión de su Eje Norte-Sur como octavo elemento, tras figurar en la lista tentativa de UNESCO desde 2013. Shanghai aspira a situarse en esta competición, a sabiendas de las limitaciones de sus recursos patrimoniales según los criterios de Valor Universal Extraordinario que aplican para las inscripciones en la LPM. No obstante, desde 2015 el gobierno de Shanghai ha hecho públicos sus planes de inclusión de su tipología residencial colonial, el Shikumen, ${ }^{1}$ dentro de la LPM. Siendo conocida la relación entre UNESCO y las grandes corporaciones turísticas globales como Tripadvisor desde 2009, no es menos relevante el papel de UNESCO en la promoción de las industrias creativas. En ese sentido, es necesario destacar cómo Shanghai es miembro desde 2010 de la Red de Ciudades

1 Significando literalmente 'puerta enmarcada en piedra', la palabra shikumen se refiere al tipo residencial especulativo producido masivamente en la etapa colonial de Shanghai, que se caracterizaba por la elaborada decoración de sus puertas de acceso, generalmente labradas en piedra. El shikumen resulta de la combinación de la casa tradicional china provista de patio y el modelo europeo de la casa adosada o row house, alcanzando entre dos y cuatro plantas de altura.
Creativas de la UNESCO como ciudad del diseño, red a la que se sumó Beijing en 2012 en los mismos términos, promoviendo eventos relacionados con la creatividad a nivel global que les ayuden a consolidar su creciente influencia.

En este escenario, el rol que juega el patrimonio construido es cada vez más relevante en términos económicos y políticos, hecho indicativo de la velocidad y envergadura a la que acontecen los cambios en China. Tras décadas de destrucción inmisericorde, la ciudad del patrimonio manifiesta una salud envidiable en China. El desarrollo económico no supone una amenaza para ella, sino que le sirve de alimento, subvirtiendo la tradicional actitud de resistencia de los preservacionistas. El patrimonio es clave en la génesis, vida y obsolescencia de la ciudad actual, y lejos de anclarse en el pasado, se ha convertido en una práctica contemporánea, auténticamente presente.

El caso de Shanghai sirve para comprobar cómo su pasado moderno se reescribe para asegurar el éxito comercial de tres pilares de la economía urbana como son la promoción inmobiliaria, el turismo y las nuevas industrias creativas. Se trata de un proceso de reconstrucción de su historia en tiempo real, que no plantea grandes contradicciones con la producción constante de narrativas en la historia moderna de China (Li, 2012), y explica su arrollador éxito reciente. Considerando a la ciudad del patrimonio en China como un excepcional campo de ensayos, la investigación sobre la nueva identidad 'moderna', o 'cosmopolita' del caso específico de Shanghai alude a la formulación de un concepto enormemente elusivo en su dimensión urbana como es la autenticidad. Ésta ha cobrado auge 
en el marketing y el turismo desde el punto de vista de la experiencia personal, y su uso en el patrimonio plantea cuestiones de calado entre las cuales se encuentra su vigencia actual (Smith, 2006); la posibilidad de plantear la conservación urbana desde un punto de vista experiencial (Guttormsen y Fageraas, 2011), o más allá, a qué grupos sociales ven privilegiada su experiencia sobre el patrimonio, en detrimento de otros menos favorecidos (Silverman, 2015).

Con el propósito de contribuir a este debate abierto, este artículo definirá en primer lugar el concepto de ciudad del patrimonio como marco operativo para entender la relación de la memoria de la ciudad contemporánea con su futuro. A continuación, caracterizará a Shanghai como ejemplo de este concepto, interpretando su cambio urbano a nivel espacial, histórico, cultural, económico y social. Por último, el artículo registrará la evolución del concepto de ciudad del patrimonio en Shanghai a la luz de iniciativas recientes que buscan la formulación de la identidad cosmopolita de Shanghai como metrópolis global. Las conclusiones del artículo valorarán la aportación de la memoria a la producción de arquitectura contemporánea en Shanghai, y sus implicaciones sociales, económicas y culturales.

\section{LA CIUDAD DEL PATRIMONIO COMO MARCO TEÓRICO CONTEMPORÁNEO}

En su libro Ciudad Hojaldre (2005), Carlos García Vázquez desarrolló un marco de referencia para la interpretación de los variados fenómenos que actualmente concurren en el modelado de la ciudad global. De manera efectiva, García Vázquez atendió al efecto físico de la cultura en el cuerpo de la ciudad en base a su adscripción a discursos disciplinares o posthistóricos, separados por concepciones diametralmente diferentes de la memoria (García Vázquez, 2005). Transcurridos doce años, es evidente cómo la historia, la cultura y la memoria ocupan un lugar cada vez más central en las narrativas del desarrollo, al servicio de la nueva economía urbana con efectos notorios en la regeneración ambiental, si bien a costa de la desposesión de sectores enteros de la sociedad (Harvey, 1989). Los estudios urbanos recientes han puesto de relieve este fenómeno, atendiendo en especial al papel de la cultura en la recuperación económica y física de la obsolescencia urbana (Zukin, 2010), y revelando su dimensión más oscura relacionada con la gentrificación. En base a la creciente importancia de las industrias culturales, la llamada 'ciudad patrimonial' es clave en la definición de políticas y en el planeamiento, condicionando la forma y el carácter de lo urbano (Fernández-Baca Casares, 1996; Ashworth, 2011).

La formulación de la ciudad del patrimonio como marco conceptual surge de reevaluar la dialéctica entre conservación y desarrollo, especialmente considerando el efecto de la crisis económica de 2008 y la ascendencia irrefrenable del patrimonio y la memoria en la política, la cultura, la economía y la sociedad de la ciudad global. Cuando recomendaciones como la del Paisaje Urbano Histórico de 2011 (UNESCO 2011; Bandarin y Van Oers, 2012), han estirado el potencial de la producción de patrimonio en el medio construido hasta sus últimos límites, la ciudad del patrimonio se convierte en una nueva categoría para caracterizar los efectos de la 'patrimonialización'. Entre ellos, la evolución de la modernidad hacia la globalización a través 
del abandono de sus aspiraciones universalistas y la recuperación de la identidad y la cultura (Liu, 1996; Dirlik, 2003), así como la influencia cuantitativa y cualitativa que la consideración al patrimonio ejerce sobre la producción arquitectónica contemporánea (Koolhaas y OteroPailos, 2014).

Esta última cuestión resulta especialmente significativa. En este sentido, nos encontramos lejos de haber explotado las posibilidades de un acuerdo entre nueva arquitectura y memoria en los términos que Aldo Rossi definió en La arquitectura de la ciudad (1971). Sobrepasando el ámbito de lo puramente disciplinar de la arquitectura y el urbanismo, la protección del patrimonio construido ha terminado designando áreas de cada vez mayor tamaño, complejidad e importancia económica y estratégica. Estas áreas constituyen uno de los sustratos fundamentales donde crece la arquitectura contemporánea, que ya no puede liberarse de los lazos patrimoniales. Y no estaremos hablando de un éxito en los términos planteados por la Tendenza: esta 'expansión ecuménica' -en palabras de Choay en 1996- de los límites del patrimonio, está vinculada a un proyecto político conservacionista global, impulsado por agentes diversos que incluyen desde la UNESCO con la lista de Patrimonio Mundial, hasta organizaciones no gubernamentales de defensa del medioambiente (Harrison, 2015).

Al contrario de la metáfora de la inacción debida al peso de los recuerdos que Borges describió en su Funes el memorioso en 1944, la patrimonialización es un proceso frenético, que muchos críticos consideran ya fuera de control
(D’Eramo, 2014). Lejos de lo que pudiera pensarse desde una perspectiva ortodoxa, el objetivo de tal proyecto no es detener, o plantear alternativas, sino fomentar el crecimiento económico y reforzar el nuevo papel del patrimonio en la gobernanza urbana contemporánea. Su resultado es la creación de lo que interpretamos como la ciudad del patrimonio, aquella que:

Es consciente de la importancia que la arquitectura y el urbanismo tienen para la competitividad entre ciudades globales (Bandarin y Van Oers, 2012).

Es activa en el propósito de crear nuevas narrativas locales y nacionales que refuercen su identidad (Ashworth and Karavatzis, 2011);

Se compromete con la cultura, la producción de nuevas tradiciones y la conservación del medio ambiente (Harvey, 1989),

Depende económicamente de los ingresos y puestos de trabajo relacionados con el patrimonio, especialmente el sector inmobiliario, las industrias culturales y el turismo (Hutton, 2004; Greffe, 2004),

Se desequilibra socialmente debido a las restricciones severas que existen en el acceso al 'derecho a la ciudad', especialmente en sus vertientes de la participación y la apropiación (Harvey, 2008).

Como ejemplificaremos en el caso de Shanghai, el desarrollo urbano reciente en China se muestra afín a la definición de ciudad del patrimonio, de la manera más literal imaginable. Tengamos en cuenta, por ejemplo, la extensa proliferación de museos arqueológicos en las periferias urbanas a lo largo del país, cuyos contenidos provienen directamente las excavaciones preventivas a las que la legislación 
patrimonial obliga para nuevas construcciones (Wertmann, 2015). El patrimonio cultural, por tanto, ha dejado de ser un obstáculo, para convertirse en un motor del desarrollo rural y urbano, haciendo buenas las palabras de Harrison (2015): 'el patrimonio tiene muy poco que ver con el pasado, sino que en realidad, incorpora prácticas que se preocupan fundamentalmente del montaje y el diseño del futuro'. Este papel activo del patrimonio se relaciona directamente con la variedad creciente de narrativas sobre la modernización en China (Dirlik, 2003; Li, 2012; Law, 2016), para las que los eventos históricos han sido tradicionalmente ensamblados y contados con el objetivo de legitimar acciones del presente (Li, 2012; Wu, 2014).

\section{SHANGHAI COMO CIUDAD DEL PATRIMONIO}

Definir a Shanghai como ciudad del patrimonio en términos absolutos pudiera parecer una osadía, especialmente por la magnitud de la destrucción patrimonial en las últimas décadas. También por la relativa desventaja que la ciudad tiene, en términos de relevancia histórica, frente a la grandes capitales imperiales de Xi'an o Beijing, reconocidas por las listas de Patrimonio Mundial de la UNESCO. Por encima de la dimensión cuantitativa, consideramos que el avance de la ola patrimonial en Shanghai ofrece una ocasión única de comprobar cómo la globalización produce 'modernidades múltiples' (Eisenstadt, 2000; Dirlik, 2003) que buscan arraigo en nuevas formulaciones, frecuentemente nostálgicas, del pasado. Los resultados del debate acerca de qué pasado elegir para el futuro de cada ciudad se manifiestan en el paisaje urbano: mientras en un contexto de nacionalismo creciente Beijing ha reforzado el discurso de la capital imperial mediante la galopante gentrificación del tejido tradicional del hutong (González Martínez, 2016), ${ }^{2}$ la apuesta de Shanghai se orienta a una recuperación decidida de su patrimonio moderno (Law, 2012; Law, 2016).

Se trata de una empresa paradójica, toda vez que la construcción de esta nueva identidad de Shanghai se traduce en el propósito elusivo de vuelta a una nueva 'tradición moderna', un regreso al futuro urbano en versión oriental. Una comprensión de la magnitud del reto requiere tener en cuenta cómo esta tradición fue completamente denigrada durante las décadas precedentes, y en especial durante la Revolución Cultural (1966-1976), por su vinculación al capitalismo. No en vano, la industrialización y proletarización de la ciudad tras la fundación de la República Popular China tuvieron como objetivo la creación de una nueva identidad obrera llamada a borrar su cosmopolitismo y la 'humillación histórica’ del colonialismo, a través de un profundo cambio social. ${ }^{3}$ Autores como Pan

2 La palabra hutong significa literalmente 'camino hacia la fuente del agua', y es la comúnmente empleada para el sistema viario en dirección Este-Oeste de la capital. Las edificaciones que se abren a los hutongs reciben el nombre de siheyuan (literalmente, patio de cuatro lados) y constituyen el tipo de edificación tradicional del espacio intramuros de Beijing.

3 A pesar de tales esfuerzos, estas políticas oficiales fueron contestadas por la continuidad de los cuadros técnicos locales de la administración semicolonial de la ciudad tras 1949, quienes perpetuaron métodos modernos de gestión urbana que fueron creados durante la época de las Concesiones. También fueron contradichas por la ciudad misma: el parque de viviendas de la época colonial fue completamente subdividido con las leyes de reforma de la propiedad de inicios de la década de 1950, si bien los cambios operaron dentro de la arquitectura y no se manifestaron de manera clara en el paisaje urbano. Por último, las infraestructuras 
y Liu (2011) han descrito cómo en el contexto de las reformas de apertura impulsadas por el presidente Deng Xiaoping en la década de 1980, la narración de la vida del Shanghai de los años '20 y'30 fue completamente renovada, compensando el olvido oficial al que fue sometida tras 1949. Ni mucho menos se trataba de un acto de justicia poética: subyaciendo en este cambio, se encontraba la oportunidad de justificar el nuevo giro económico en una razón histórica, como era la recuperación de la 'esencia olvidada', por supuesto capitalista, de Shanghai. La recuperación de esta memoria va de la mano de un cambio radical dictado nuevamente por las políticas estatales, que han determinado el desmantelamiento del otrora poderoso tejido industrial de Shanghai y la terciarización de su economía.

Proponemos describir este proceso de recuperación de la identidad a través de cinco actos, en los que la arquitectura aparezca de manera instrumental, confirmando su condición de herramienta del cambio espacial, histórico, cultural, económico y social.

\section{PRIMER ACTO. LA BÚSQUEDA DE UN IMPACTO GLOBAL}

Especialmente tras el mítico 'Viaje al Sur' del presidente Deng en 1992, que marcó el resurgir de Shanghai como capital económica de China, decisiones políticas y de planeamiento como el desarrollo del centro financiero de Lujiazui en el distrito de Pudong tuvieron como objetivo la producción de una identidad de impacto para la ciudad. El concurso de diseño urbano de Lu-

de la ciudad, las más avanzadas de Asia de la época, continuaron su vida operativa sin necesidad de grandes actualizaciones hasta entrados los 1980. jiazui, desarrollado a continuación de este pistoletazo de salida, fue en sí la puesta de largo de China en el escenario de las competiciones internacionales en esa década prodigiosa que fueron los años 90 para la arquitectura global del fin de la historia (Xue, 2006). El relato del concurso es explicativo de la importancia del nuevo discurso simbólico: el diseño vencedor de Richard Rogers, que multiplicaba la densidad del área para fomentar la movilidad peatonal como nuevo Manhattan, fue finalmente abandonado para favorecer la composición de grandes ejes de inspiración francesa del consorcio sino-francés para la planificación del Pudong que desarrolló finalmente el proyecto, y que planteó, al igual que en la Défense de París, un modelo urbano de torres dispersas, completamente dependiente del automóvil.

La referencia al pasado colonial francés de la ciudad dejaba de ser humillante, para convertirse en guía del desarrollo. Los mismos hitos, reinterpretados, hacían un viaje de ida y vuelta, y la Perla de Oriente, nueva torre de televisión construida en el extremo de la península, reprodujo la silueta ascendente de la Torre Eiffel, si bien doblándola en altura. Más allá de las conexiones imaginadas, la finalización de la torre en 1995 confirmó el potencial escenográfico de Lujiazui para la construcción en altura (Rowe, 2011). El nuevo y pintoresco skyline levantado en los últimos 25 años en la margen derecha del rio Huangpu ha equilibrado y ofrecido continuidad simbólica a la fachada del Bund, el paseo fluvial que hasta entonces había sido imagen del Shanghai semicolonial y capitalista (Hubbert, 2017). Al mismo tiempo, y de manera que interesa especialmente a nuestro discurso, se inició entonces la carrera 


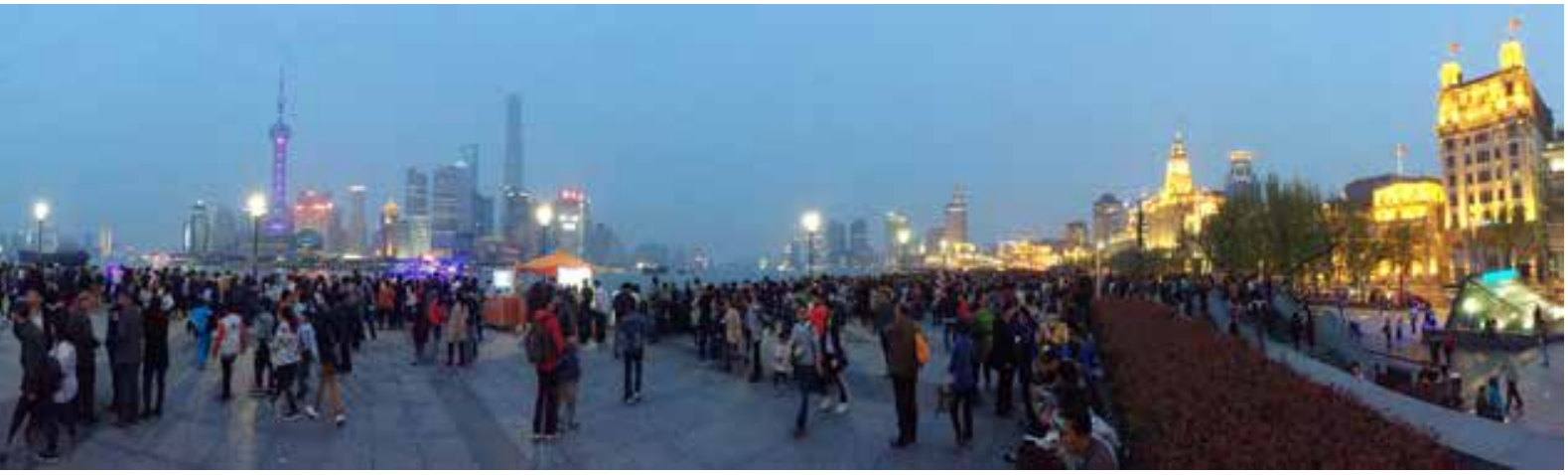

Figura 1. Rascacielos históricos del Bund de Shanghai (derecha), con el nuevo distrito financiero de Lujiazui (izquierda). Fuente: Plácido González Martínez, 2017.

por la protección del patrimonio construido de Shanghai, que cuenta con un hito de referencia en la recuperación de los rascacielos históricos del Bund: el 'Viejo Shanghai' ha sido elevado a los altares de la historia de la arquitectura del siglo XX como ciudad del Art Deco, completando un triángulo que la une a París y Nueva York, las otras dos grandes capitales artísticas y económicas de Occidente, de manera intencionadamente simbólica.

\section{SEGUNDO ACTO. LA RELACIÓN CON LA MEMORIA}

El desarrollo de una variante shanghainesa de la modernidad históricamente legitima sus aspiraciones a ocupar un papel protagonista entre los nuevos centros del capitalismo en Asia y a nivel mundial (Law, 2012; Law, 2016). En términos prácticos, esto convierte al socialismo en una etapa pasada de la historia de la ciudad, que evoluciona asumiendo las tesis económicas del capitalismo en el marco de una economía plani- ficada (Liu, 1996; Dirlik, 2016). Las consecuencias urbanas de este hecho pueden comprobarse en el borrado de la memoria del trabajo en la ciudad, que en términos patrimoniales ortodoxos, podemos calificar de perfecto falso histórico. Especialmente significativa en este sentido es la alianza que este proceso ha encontrado con el auge de las industrias culturales y creativas, paralelo a una rápida obsolescencia industrial que ha librado las márgenes del río Huangpu y sus afluentes, como el Suzhou Creek, para un nuevo disfrute ocioso de los espacios que anteriormente conformaban el corazón proletario de la ciudad.

Los últimos Planes Quinquenales, tanto a nivel estatal como municipal, han promovido esta nueva economía de manera decidida, encontrando en el patrimonio industrial un marco óptimo de desarrollo. De manera completamente experimental, las intervenciones de recuperación del patrimonio urbano ensayan formas de gestión mixta, que disponen de las preexistencias para armar un nuevo discurso histórico. Como ejemplo, el nuevo conjun- 


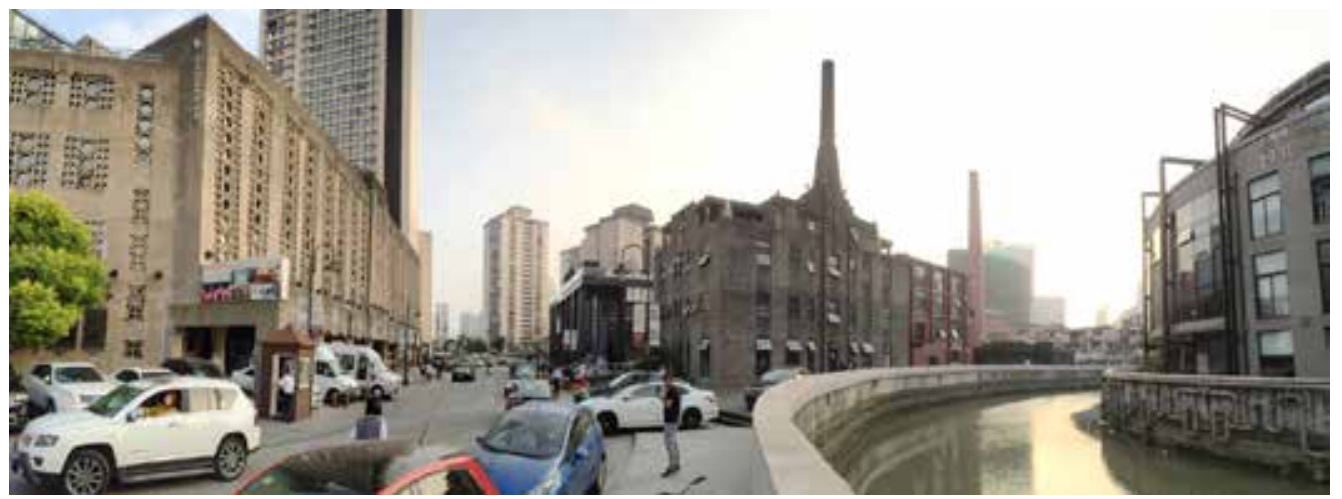

Figura 2. El antiguo matadero de Shanghai (1933), rehabilitado como edificio emblema del Shanghai Music Valley en el distrito de Hongkou. Fuente: Plácido González Martínez, 2017.

to denominado Shanghai Music Valley en el distrito de Hongkou es representativo de esta tendencia (González Martínez, 2017). Barrio histórico de Shanghai situado en la antigua concesión japonesa, la intervención desde 2012 de una empresa de gestión patrimonial ha llenado sus calles de carteles que evocan pasados imaginarios para nuevos edificios; reescribiendo el patrimonio existente para cancelar todo tipo de asociaciones con su pasado anterior, empleando la arquitectura como carcasa más o menos pintoresca que enmarca todo tipo de actividades económicas al servicio de la sociedad de consumo.

El ansia por una nueva historia se lleva asimismo a la periferia de la ciudad. La suburbanización galopante de Shanghai en los últimos 10 años ha resultado en el ensayo de nuevas medidas de 'reidentificación'. Las intervenciones desarrolladas en la red de 'Ciudades de Agua' que rodean Shanghai han ido encaminadas a dar marcha atrás a la historia; como es el caso de Zhenzhe, donde se está llevando a cabo la reexcavación de los canales históricos de la ciudad, cegados durante la Revolución Cultural, dentro de una estrategia encaminada a su inscripción en la LPM de UNESCO. Esta marcha atrás en la historia se acompaña del increíble tránsito de edificaciones patrimoniales: viviendas, juzgados, mercados de las dinastías Ming y Qing, que son desmontadas en las provincias vecinas de Jiangsu y Anhui, para reconstruirse como nuevos hoteles de lujo en las periferias anónimas de ciudades satélite como Zhujiajiao y Shaoxing.

\section{TERCER ACTO. UNA CULTURA COSMOPOLITA CONSOLIDADA}

La cultura haipai (literalmente traducida como cosmopolita) de Shanghai ha sido valorada como constitutiva del carácter de la ciudad, y la incorporación del imaginario moderno ha sido constante en el cine y las artes visuales (Lagerqvist, 2006). Un recuento muestra cómo de las 13 películas nacionales e internacionales ambientadas en Shanghai que fueron producidas desde 1984 hasta 2012; siete se localizan temporalmente en 
las décadas de 1920, 30 y 40 (Teo, 2017). En este sentido hemos de señalar la imposición de la estética asociada a esta época en la gentrificación gradual de los antiguos shikumen de los distritos centrales de Shanghai, en una suerte de reoccidentalización tras décadas de tugurización y ocupación por parte de los sectores económicamente más desfavorecidos de la sociedad. De igual manera, la occidentalización de la imagen de Shanghai ha sido un propósito presente en la construcción de la periferia, ejemplificada en el proyecto 'Shanghai 9 Towns' (Den Hartog, 2011), que significó la construcción de nueve ciudades, cada una con su propio estilo característico -entre ellas, el holandés (Gaoqiao), inglés (Songjiang), alemán (Anting), italiano (Pujiang)- recuperando la ilusión temática de un Shanghai diverso, aunque con sus ingredientes convenientemente separados. ${ }^{4}$

Existe consenso al señalar a la Exposición Mundial de 2010 como piedra de toque de esta nueva revolución cultural. El lema de la muestra, 'Better City, Better Life', fue claramente explicativo de la confianza que se depositaba en la ciudad como soporte, y de la calidad de su patrimonio construido como aglutinador de la nueva identidad. Lo cual no dejó de ser paradójico: autores diversos han señalado cómo desde el gobierno estatal hasta las instituciones municipales organizadoras de la Exposición, existió la voluntad firme de ensayar en Shanghai el modelo de ciudad sin infravivienda que actualmente se aplica como objetivo de las políticas urbanas en China (Wong y Liu, 2016). El coste que implicó, con la demolición de cer-

\footnotetext{
4 Sirva de anécdota señalar que la construcción de la nueva ciudad española fue cancelada a causa de un escándalo de sobornos, y su promotor condenado por corrupción.
}

ca de 60 millones de $\mathrm{m} 2$ de tejido residencial obsoleto en el centro de la ciudad, y el desplazamiento a las periferias de 1.200 .000 hogares (He y Wu, 2005), fue asumido como sacrificio necesario, y significó el avance de la llamada gentrificación de nueva construcción en el corazón de la ciudad, incorporando nuevas clases profesionales, de gustos avanzados y con poder adquisitivo elevado.

Éstos son los consumidores preferentes de un sector de la cultura de importancia creciente en la economía de la ciudad. En este sentido, la política juega con dos barajas: por un lado, reforzando sus lazos históricos con otros grandes centros artísticos internacionales como son París, Londres y Nueva York, y evitando al mismo tiempo deliberadamente la prolongación franquiciada del dominio cultural de Occidente. Esto no es óbice para que la inspiración, formal e institucional, de centros de referencia como la Tate Modern londinense estuviese presente en la creación del Power Station of Art en una antigua central eléctrica a orillas del Huangpu; o que iniciativas de recuperación de las márgenes del mismo río vengan apoyadas por la construcción de nuevos centros de arte como el Long $\mathrm{Mu}$ seum y los Silos de la Shanghai Urban Space Art Season, obras ambas del estudio local Atelier Deshaus; o de arquitectos japoneses como Sou Fujimoto, autor del Yuz Museum.

\section{CUARTO ACTO. LA RENTABILIDAD DEL RECICLAJE}

La dimensión económica del patrimonio se revela, en primer lugar, en el marco del mercado inmobiliario, que constituye la principal fuente de ingresos del gobierno municipal y de los 
distritos. La conservación de patrimonio forma parte de un complejo equilibrio económico que motiva su rentabilización, y por tanto, su comercialización (He y Wu, 2005). En paralelo, es necesario señalar cómo en el ascenso de las industrias creativas en Shanghai, la ciudad está amortizando el valor patrimonial de antiguos conjuntos industriales como emplazamiento óptimo de nuevas empresas. Este es el caso de la iniciativa pionera del M50, que arrancó en 2000 promovida por el artista Xue Song, para congregar en una fábrica abandonada a orillas del Suzhou Creek a una comunidad de artistas atraídos por el bajo coste del alquiler y las dimensiones de los espacios. Su evolución posterior ejemplifica las tensiones alrededor de sector cultural en China: por un lado, el control gubernamental sobre la producción artística obligó a que esta asociación inicialmente libre de creadores se aviniese a un funcionamiento reglado y participado por las autoridades. A cambio, el nuevo apoyo económico ofrecido por las instituciones ha terminado convirtiendo a M50 en una franquicia de espacios de producción artística, que cuenta con sedes repartidas en otras áreas centrales de la ciudad (Wang, 2009).

Al mismo tiempo, Shanghai es motor el desarrollo del turismo urbano en China. La iniciativa actualmente en curso de proponer la tipología residencial del shikumen como patrimonio mundial de la UNESCO es una clara muestra de este perfil, reforzada en la periferia con el proyecto anteriormente mencionado de inclusión en la LPM del conjunto de nueve Ciudades de Agua. Orientadas hacia el mercado global, la participación del World Heritage Institute of Training and Research (WHITRAP) en su recuperación es una muestra clara de la implicación de la propia UNESCO en su promoción. Junto a esta dimensión global del turismo, Shanghai cuenta con un atractivo nacional específico, como es la vinculación histórica de la ciudad a los orígenes del Partido Comunista Chino. Éste es un valor fundamental en el auge del llamado 'turismo rojo' orientado al mercado nacional, que la hace rivalizar en importancia con otras ciudades de enorme significación política como Beijing o Nanjing.

Esta dinámica no está exenta de lo que a ojos occidentales aparecen como paradojas, como es el hecho de que el principal destino del 'turismo rojo' de la ciudad, la sede del Primer Congreso del Partido Comunista Chino, se encuentre situado en el corazón de la gentrificada área de Xintiandi. Localizada en una de las zonas históricamente más deprimidas de la antigua concesión francesa de la ciudad, fue un promotor de Hong Kong, el grupo Shui On, el que aprovechó el programa de demoliciones diseñado por el gobierno de Shanghai en 1996 y conocido como 'Plan 365' (implicaba la desaparición de 365 hectáreas en áreas centrales de la ciudad), para acordar con las autoridades la 'conservación' de la manzana de shikumen en la que se encontraba este lugar histórico según el proyecto regulador diseñado por la oficina estadounidense SOM. Para horror de conservacionistas occidentales, tras ser documentada, esta manzana histórica fue reconstruida para albergar el que es hoy uno de los centros turísticos más vibrantes de la ciudad. Al norte de Xintiandi, otro lugar de significación histórica y visita obligada de los 'turistas rojos' es la residencia de Mao Zedong en los años 20 en Shanghai. En este caso, el acuerdo con los promotores no consideró la conservación de la 


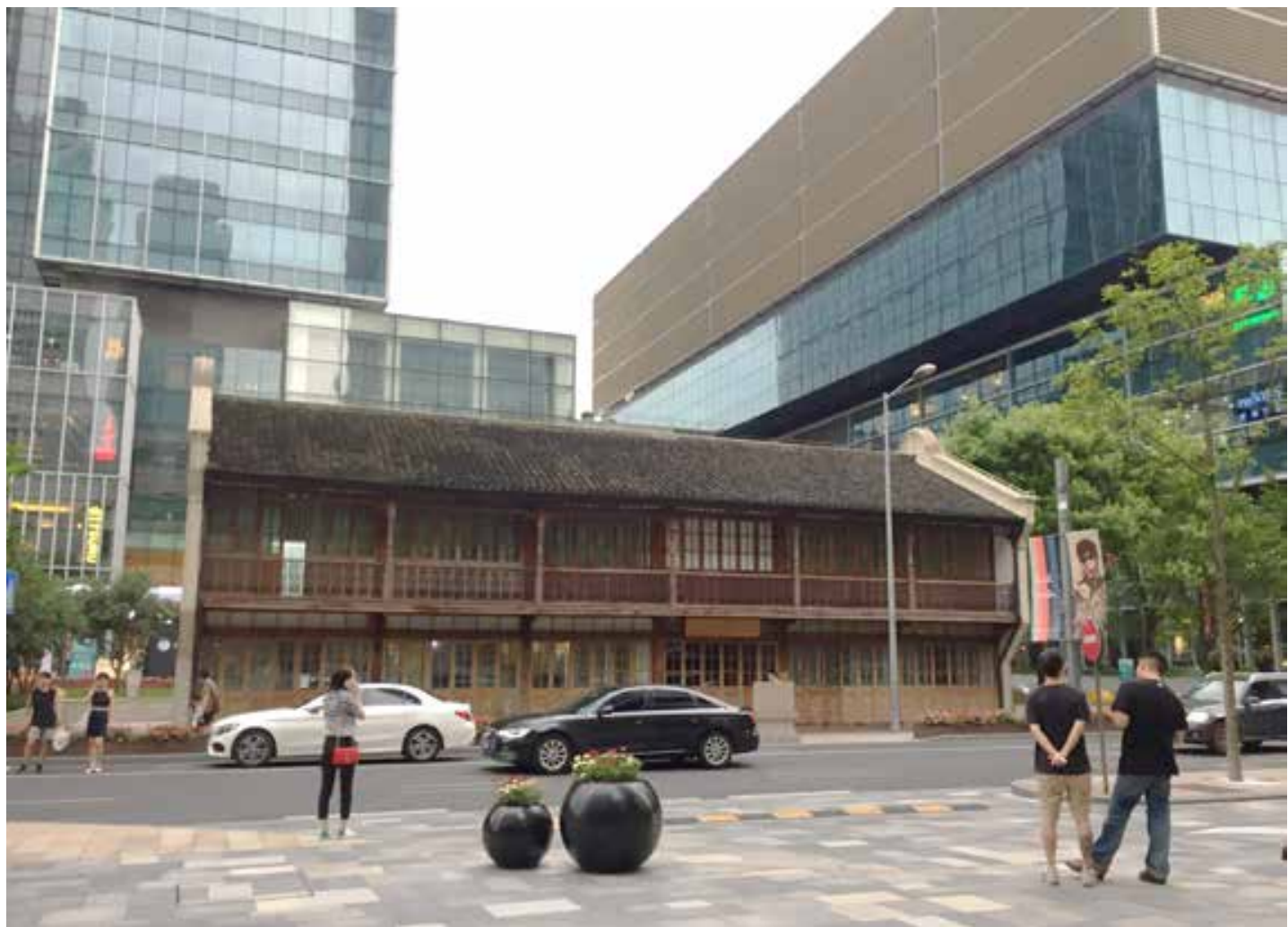

Figura 3. Antigua residencia de Mao Zedong en Shanghai, rodeada por los rascacielos del área comercial de Jing’An. Fuente: Plácido González Martínez, 2017.

manzana: de manera de nuevo heterodoxa para los cánones occidentales, el plan solamente respetó el edificio de dos plantas de altura, que se yergue actualmente aislado en un vacío entre los grandes rascacielos de la zona de Jing'An.

\section{QUINTO ACTO. EL AUTÉNTICO DERECHO A LA CIUDAD}

En el contexto de la nueva economía, la gestión empresarial del patrimonio a través de conglomerados público-privados (González Martínez, 2017) pone actualmente en riesgo su dimensión social, que todas las convenciones recientes consideran componente fundamental de su autenticidad. A este respecto, Shanghai está siguiendo una tendencia internacional, para la cual la referencia de Xintiandi se ha extendido a otras ciudades chinas, en un proceso de 'Xintiandización' que es análogo al 'Efecto Guggenheim' en el ámbito del patrimonio en China. Confiando en los efectos sanadores de la recuperación del patrimonio residencial tradicional para usos exclusivamente comerciales, este proceso de gentrificación comercial se extiende por la ciudad a pesar del alto volumen de expulsiones y relocalizaciones a las que ha obligado. 
Esto ha llevado al fracaso de la experimentación en modelos alternativos de gestión del patrimonio para el patrimonio residencial de Shanghai, los shikumen, que contesten la imposición de discursos patrimoniales autorizados a través del fomento de la participación y la corresponsabilidad de los residentes. Este fue el caso del barrio de Tianzifang, ${ }^{5}$ en el que la participación de los residentes en la génesis y gestión del barrio rompió completamente con prácticas ortodoxas establecidas, y cuyo excepcional resultado ha sido difícilmente reproducible en otras áreas de la ciudad o de la propia China (Wang, 2011). De igual manera, la intervención por parte de las autoridades de distrito en la rehabilitación del conjunto de Bugaoli permitió la actualización de las infraestructuras obsoletas de las viviendas y el mantenimiento de la población original, siguiendo el modelo de rehabilitación de barrios que durante la década de los 90 se puso en práctica en Europa. Pero una vez concluida, el altísimo coste de la experiencia de Bugaoli la ha convertido en experiencia excepcional, inviable para su aplicación a otros barrios según el actual modelo de gestión.

Estos casos nos devuelven a la dimensión social del patrimonio, vinculada a una discusión aún más profunda, y relativa a la autenticidad. En este sentido, las definiciones de lo auténtico que se aplican actualmente de manera generalizada al patrimonio construido, arquitectónico y urbano, vienen determinadas por el discurso de la experiencia. Éste localiza

5 La renovación de Tianzifang consistió en la transformación de un antiguo área de shikumen en espacios de creación artística por iniciativa de artistas y residentes locales. Aunque sigue albergando a parte de la población original, actualmente es un enclave turístico en proceso de gentrificación comercial galopante, de gran éxito popular. la raíz del patrimonio no ya en los propios objetos patrimoniales, sino en la subjetividad de dos modelos fundamentales de usuarios devenidos en consumidores: el del turista y el del nuevo profesional creativo. La cuestión pendiente es, por tanto, la ampliación del mismo discurso de la experiencia a todos los sectores de la sociedad, lo cual atañe directamente a la definición y límites del derecho a la ciudad. Su interpretación desde un punto de vista occidental queda en entredicho por el propio marco legal y la interpretación de la filosofía del derecho en China (Shin, 2013; Wong y Liu, 2016). Para esta cuestión, entendiendo al patrimonio como motor y recurso económico, cultural, espacial y social, se comprueban las carencias del modelo de Shanghai: una vez que experiencias alternativas como M50 o Tianzifang fueron 'normalizadas' a través del control en la gestión y la gentrificación, la participación de la población en la identificación, uso y transformación del patrimonio es inexistente, como también lo es en el reparto y disfrute de sus beneficios, lo que redunda en una creciente segregación espacial.

\section{CONCLUSIÓN}

La definición de Shanghai como ciudad del patrimonio implica un refuerzo de la búsqueda de identidad a través de diferentes vías. Las más tradicionales, vinculadas a la protección patrimonial, muestran un desarrollo acelerado, siempre con retraso respecto a una frenética destrucción del tejido urbano histórico iniciada a mediados de la década de 1990 y que, transcurridos más de veinte años, aparentemente comienza a remitir. Además del interés político por la construcción de una narrativa propia, el principal motivo de este propósito es la inmedia- 
ta capitalización de la memoria por parte de la industria inmobiliaria y del turismo, que dejan de lado cualquier tipo de consideración social.

Shanghai aparece como laboratorio en el que testar la coexistencia de referencias históricas múltiples, en un marco cultural para el que la historia tradicionalmente ha sido un proceso de ensamblaje, en el que la autenticidad del relato queda en manos de la verificable autenticidad de los fragmentos (Callahan, 2015). La definición de la identidad de Shanghai como ciudad del patrimonio se basa fundamentalmente en la visión desde fuera, presente en la ciudad desde los inicios de su historia colonial en 1842, y clave para la apropiación de la cultura moderna importada por las potencias que ocuparon sus concesiones.

Autores como Chang (2017) inciden en resaltar el doble filo de esta visión; cosmopolita y abierta al cambio, aunque provinciana en la preferencia acrítica por lo foráneo. Si bien los estudios culturales sobre Shanghai ha insistido fundamentalmente en la vinculación de su arquitectura al Art Deco y a su urbanismo con la verticalidad del Bund y Luijiazui como manifestaciones de futurismo, los ejemplos presentados muestran el lado menos explorado y controvertido desde una perspectiva local, como es la influencia de la visión exterior en definir la relación de Shanghai; no ya con su futuro, sino con su propio pasado.

Esta ciudad de crecimiento vertiginoso, que ha vivido en los últimos treinta años un proceso de reforma solamente comparable al experimentado por París durante la era Haussmann, sigue reclamando la búsqueda de su carácter al término del túnel del desarrollo, lo que descarta cualquier definición apriorística y ortodoxa de qué ha de ser auténticamente Shanghai. Una identidad escapadiza y elusiva, que se ensambla y desmadeja de manera continua, sin principio ni final.

Bordieu, P. (1986). The form of capital. En J. Richardson (Ed.). Handbook of theory and research in the sociology of education (pp. 241-258). Westpot; CT: Greenwood Press.

Callahan, W.A. (2015). History, tradition and the China Dream: socialist modernization in the World of Great Harmony. Journal of Contemporary China 24:96, 983-1001

Chang, Q. (2017). A Chinese approach to urban heritage conservation and inheritance: focus on the contemporary changes of Shanghai's historic spaces. Built Heritage 3, 65-87. 
D’Eramo, M. (2014). Unescocide. New Left Review $88,47-53$.

Den Hartog, H. (2011). Shanghai New Towns: Searching for Community and Identity in a Sprawling Metropolis. Rotterdam: 010 Publishers.

Dirlik, A. (2003). Global modernity? Modernity in an age of global capitalism. European Journal of Social Theory 6 (3), 275-292.

Dirlik, A. (2016). Modernity and revolution in Eastern Asia: Chinese socialism in regional perspective. Translocal Chinese: East Asian Perspectives 10, 13-32.

Eisenstadt, S.N. (2000). Multiple modernities. Daedalus 129 (1), 1-29.

Fernández-Baca Casares, R. (1996). La ciudad patrimonial. Boletin PH 14, 88-95

García Vázquez, C. (2005). Ciudad hojaldre. Visiones urbanas del siglo XXI. Barcelona: Gustavo Gili.

González Martínez, P. (2016). Authenticity as a challenge in the transformation of Beijing's urban heritage: The commercial gentrification of the Guozijian historic area. Cities 59, 48-56.

González Martínez, P. (2017). Urban authenticity at stake: A new framework for its definition from the perspective of heritage at the Shanghai Music Valley. Cities 70, 55-64

Greffe, X. (2004). Is heritage an asset or a liability? Journal of Cultural Heritage 5 , 301-309.
Guttormsen, F.S.; Fageraas, K. (2011). The social production of 'attractive authenticity' at the World Heritage Site of Roros, Norway. International Journal of Heritage Studies 17 (5), 442-462.

Harrison, R. (2015). Beyond 'natural' and 'cultural' heritage: towards an ontological politics of heritage in the age of Anthropocene. Heritage and Society 8 (1), 24-42.

Harvey, D. (1989). From managerialism to entrepreneurialism: the transformation of urban governance in late capitalism. Geografiska Annaler Series B Human Geography 71 (1), 3-17.

Harvey, D. (2008). The right to the city. New Left Review 53, 23-40.

He, S.; Wu, F. (2005) Property-led redevelopment in post-reform China: A case study of Xintiandi redevelopment project in Shanghai. Journal of Urban Affairs 27 (1), 1-23.

Hubbert, J. (2017). Back to the future: the politics of culture at the Shanghai Expo. International Journal of Cultural Studies 20 (1), 48-64.

Hutton, T. (2004). The new economy of the inner city. Cities 21 (2), 89-104.

Koolhaas, R.; Otero-Pailos, J. (2014). Preservation is overtaking us. New York: GSAPP Books.

Lagerqvist, A. (2006). Future lost and resumed: media and the spatialization of time in Shanghai. Paperfrom the ESF-LiU Conference "Cities and Media: Cultural Perspectives on Urban Identities in a Mediatized World", Valdstena 25-29 October 2006. 
Law, A. (2012). Postcolonial Shanghai; an urban discourse of prosperity and futurity. In Rajagopalan, M.; Desai, M (Eds.) Colonial Frames, Nationalist Histories: Imperial Legacies, Architecture, and Modernity. London: Routledge, 285-305.

Law, A.M. (2016). Searching for "New Modernities" and constructing Chinese modern identities: using history and heritage in the indigenization of global capitalist modernity in Chinese cities. Proceedings of the Heritage of China International Symposium, Xi'an Jiaotong University, Suzhou, April 2016.

Li, W. (2012). Reinventing China: Imagination and Authenticity in Chinese Historical Writing. Honolulu: University of Hawaii Press.

Liu, K. (1996). Is there an alternative to (capitalist) globalization? The debate about modernity in China. Boundary 23 (3), 193-218.

Pan, T., Liu, Z.J. (2011). Place matters. An ethnographic perspective on historical memory, place attachment and neighborhood gentrification in postreform Shanghai. Chinese Sociology and Anthropology 43 (4), 52-73.

Ren, X., Sun, M. (2012). Artistic urbanization: creative industries and creative control in Beijing. International Journal of Urban Research, 36(3), 504-521

Rossi, A. (1971). La arquitectura de la ciudad. Barcelona: Gustavo Gili, 1971.

Rossiter, N. (2006). Creative industries in Beijing. Initial thoughts. Leonardo 39(4), 367-370
Rowe, P. (2011). Emerging architectural territories in East Asian cities. Basel: Birkhaüser.

Sassen, S. (1991, 2002). The Global City: New York, London, Tokyo. Princeton: Princeton University Press.

Shin, H.B. (2013). The right to the city and critical reflections on China's property right activism. Antipode 45 (5), 1167-1189.

Silverman, H. (2015). Heritage and authenticity. The Palgrave Handbook of Contemporary Heritage Research. London: Palgrave Mac Millan.

Smith. L. (2006). Uses of heritage. Oxford: Routledge.

Teo, H.M. (2017). Palimpsests of the 'Lonely Island': Wartime Shanghai in the Western Literary Imagination. Paper from the Conference "Modernisms and Modernities East, West and South: comparing literary and Cultural Experiences”, Fudan University, Shanghai, July 2017.

Timberlake, M., Wei, Y.D., Ma, X., Hao, J. (2014). Global cities with Chinese characteristics. Cities, 41, 162-170.

UNESCO (2011). Recommendation on the Historic Urban Landscape. [Online] <http://whc. unesco.org/en/activities/638> [Accessed 6.3.2016]

Wang, S.W.H. (2011). Commercial gentrification and entrepreneurial governance in Shanghai: A case study of Taikang Road Creative Cluster. Urban Policy and Research 29 (4), 363-380 
Wang, J., Lau, S.S.Y. (2009). Gentrification and Shanghai's new middle class: another reflection on the cultural consumption thesis. Cities 26, 57-66

Wertmann, P. (2015). Popularisierung von Archäologie und Kulturerbe in China. e-Forschungsberichte des DAI, 3, 19-28.

Wong, T.C., Liu, R. (2016). Developmental urbanism, city image branding and the "Right to the city" in transitional China. Urban Policy and Research.

Wu, Z. (2014). Let fragments speak for themselves: vernacular heritage, emptiness and Confucian discourse of narrating the past. International Journal of Heritage Studies 20 (7-8), 851-865.

Xue, C.Q.L. (2006). Building a Revolution: Chinese Architecture since 1980. Hong Kong: Hong Kong University Press.

Yulong, S., Hammett, C. (2002). The potential and prospect for global cities in China: in the context of the world system. Geoforum, 33, 121-135.

Zukin, S. (2010). Naked City. The Death and Life of Authentic Urban Places. New York: Oxford University Press.

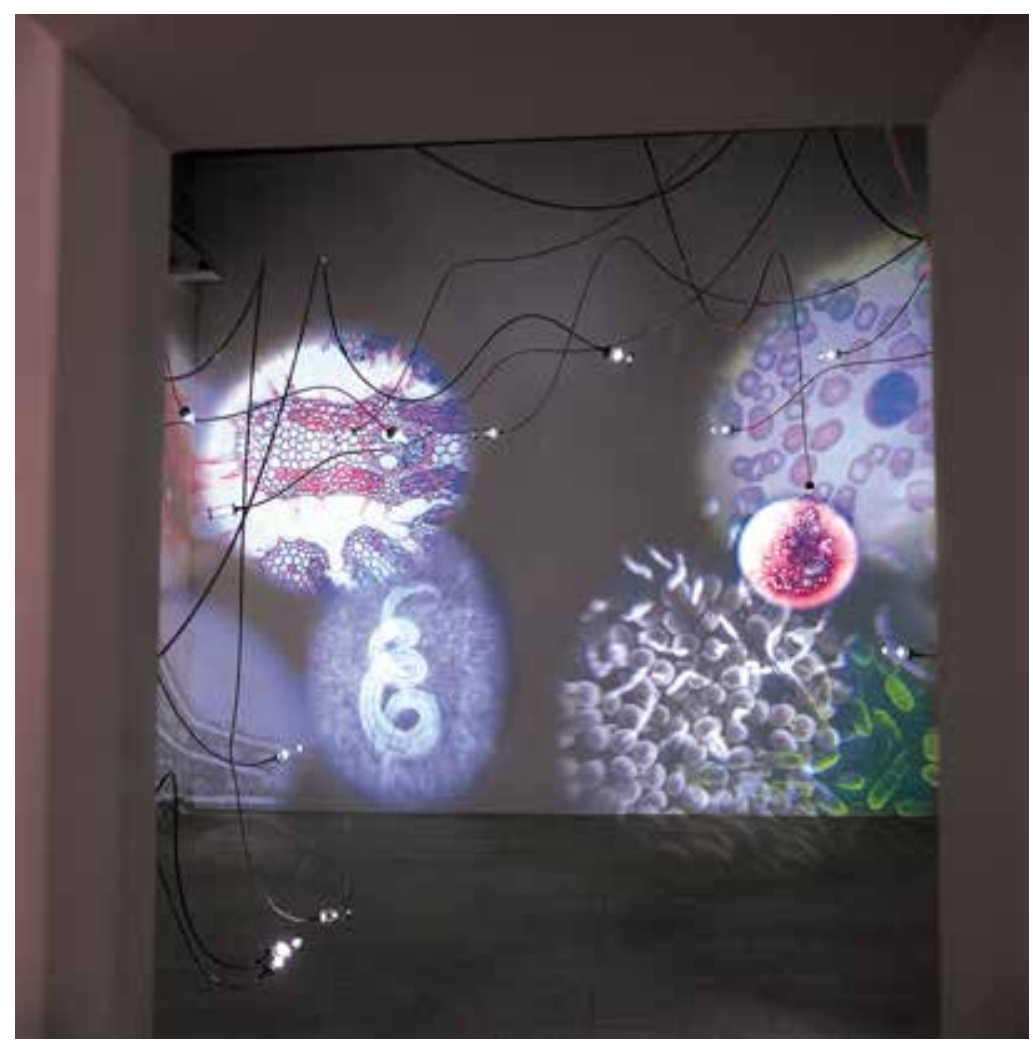

